\title{
Poster Summaries
}

Arcavi, I.

Belanger, G.

Chelouche, D.

Chernenko, A.

Copperwheat, C., Marsh, T., Dhillon, V, Littlefair, S. \& Steeghs, D.

Davenport, J. et al.

Hakala, P., Ramsay, G. \& Barclay, T.

Hancock, P., Gaensler, B. \& Murphy, T.

Hilton, E. J., Hawley, S. L. \& Kowalski, A. F.

Jones, D.

Kains, N., Browne, P., Horne, K. \& Cassan, A.

Karpov, S. et al.

King, O. G.

Kondratiev, V. I. \& Bilous, A. V.

Konidaris, N., Ben-Ami, S., Quimby, R. \& Kulkarni, S

Larsson, S., Ciprini, S., Escande, L., Lott, B. \& Madejski, G.

Levitan, D.

Lodato, G.

Manchanda, R. K.

Miller, A. A., Bloom, J. S. \& Butler, N. R.

Moriya, T.

Morokuma, T. et al.

Nugent, P.

Pekeur, N., Potter, S. B., Chadwick, P. M. \& Daniel, M.

Plavchan, P. et al.

Ramsay, G. \& Barclay, T.

Rickett, B., Coles, B., Stinebring, D. \& Gao, J. J. 442

Ridgway, S. \& Chandrasekharan, S.

Rimoldini, L. et al.

Roming, P. 
Shibahashi, H. \& Naito, J.

Shin, M.-S.

Siemion, A \& Werthimer, D.

Singer, L. \& Fotopoulos, N. 446

Sivakoff, G. R. $\quad 446$

Steele. I. A. $\quad 447$

Tang, S., Grindlay, J., Los, E. \& Servillat, M. 447

Terron, V., Fernandez, M. \& Petr-Gotzens, M. G. 448

$\begin{array}{ll}\text { Tominaga, N. } & 448\end{array}$

Tsang, D., Read, J., Hinderer, T. \& Piro, T. 449

Turner, D. G. et al. 449

Van Velzen, S., Koerding, E. \& Falcke, H. 450

Wallace, P. $\quad 450$

Williams, P. K. G. \& Bower, G. C. 450

Woudt, P. \& Fender, R. 451

Wraight, K. T., White, G. J., Bewsher, D. \& Norton, A. J. 451

Yaron, O., Gal-Yam, A. et al. $\quad 452$

Yaron, O., Gal-Yam, A. et al. 452

$\begin{array}{ll}\text { Zauderer, B. A. } & 453\end{array}$ 